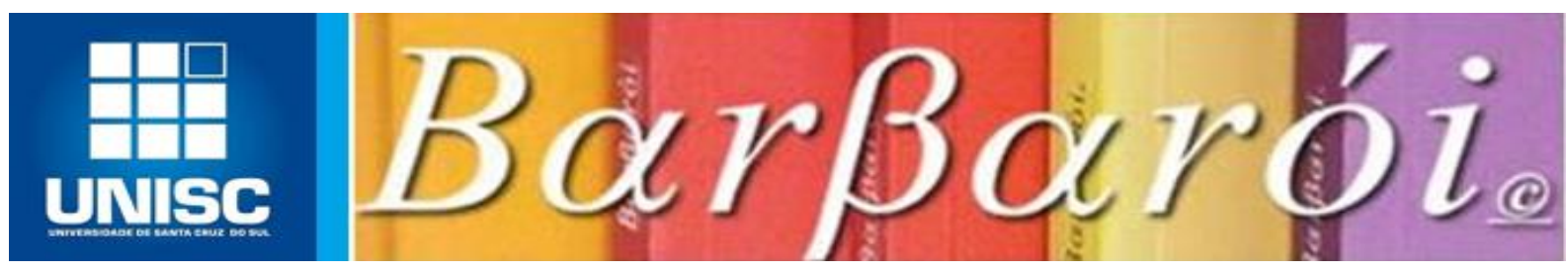

\title{
ACONSELHAMENTO PSICOLÓGICO E PSICOLOGIA POSITIVA NA SAÚDE PÚBLICA: ESCUTA COMO PRODUÇÃO DE SAÚDE
}

DOI: http://dx.doi.org/10.17058/barbaroi.v0i0.4991

\author{
$*$ \\ Fabio Scorsolini-Comin \\ Escola de Enfermagem de Ribeirão Preto da Universidade de São Paulo - EERP-USP - Brasil
}

\section{Resumo:}

O objetivo deste estudo teórico é apresentar e discutir o aconselhamento psicológico no campo da saúde pública como forma de proporcionar uma escuta enquanto produção de saúde, ou seja, partindo do pressuposto de que o espaço criado para a fala acerca dos problemas vivenciados já constitui uma ferramenta capaz de produzir bem-estar. A Psicologia Positiva é trazida como abordagem adequada aos pressupostos da saúde pública e do aconselhamento por privilegiar o domínio da saúde na compreensão do ser humano, suas virtudes e potencialidades. Refletindo sobre a formação profissional em Psicologia no campo da saúde pública, o aconselhamento é apresentado também como possibilidade de inserção do estagiário neste cenário e de aprendizagem significativa acerca das competências e habilidades que devem atravessar e constituir o ser profissional. Conclui-se que a escuta pode ser valorizada pelos profissionais de saúde como forma de aproximá-los dos clientes e potencializar o desenvolvimento e o reconhecimento de recursos para a promoção do bemestar.

Palavras-chave: aconselhamento psicológico; saúde pública; Psicologia Positiva.

\section{Introdução}

Ao longo dos anos, o aconselhamento psicológico tem sido uma das estratégias mais utilizadas no campo da saúde como forma de proporcionar acesso a informações e, ao mesmo tempo, um espaço para a expressão de sentimentos e dificuldades no domínio dos processos de saúde-doença. Assim, é reconhecido tanto como uma prática profissional em saúde como um campo de pesquisa acerca da relação de ajuda estabelecida entre profissionais e clientes, das ferramentas de comunicação e suas potencialidades em termos do acompanhamento de tratamentos e, mais recentemente, em seu viés educativo voltado à promoção da saúde. Tratase, portanto, de uma estratégia de prevenção em saúde pública que atua no âmbito do indivíduo, que deve ser acompanhado no processo de reconhecer riscos à sua saúde, bem como refletir sobre medidas preventivas e que promovam o bem-estar (MORENO; REIS, 2013; PEQUENO; MACÊDO; MIRANDA, 2013).

O objetivo deste estudo teórico é apresentar e discutir o aconselhamento psicológico no campo da saúde pública como forma de proporcionar uma escuta enquanto produção de 
saúde, ou seja, partindo do pressuposto de que o espaço criado para a fala acerca dos problemas vivenciados já constitui uma ferramenta capaz de produzir bem-estar e, portanto, saúde. Para tanto, iniciamos por explicitar e definir o aconselhamento empregado na saúde, bem como seu diálogo com diferentes disciplinas e saberes. Posteriormente, aproximaremos esse conceito dos pressupostos da saúde pública, notadamente no contexto brasileiro e tendo como referência o Sistema Único de Saúde (SUS), seus equipamentos, orientações e modelos de atenção.

Na parte final, traremos algumas propostas emergentes no campo da saúde pública a partir da perspectiva teórica e metodológica da Psicologia Positiva, considerada uma abordagem que privilegia o domínio da saúde na compreensão do ser humano, suas virtudes e potencialidades. Refletindo sobre a formação profissional em Psicologia no campo da saúde pública, o aconselhamento é apresentado como recurso não apenas para a atuação futura, por exemplo, em equipamentos de saúde, como possibilidade de inserção do estagiário neste cenário e de aprendizagem significativa acerca das competências e habilidades que devem atravessar e constituir a formação desses profissionais, ou seja, da valorização da escuta também como produtora de saúde e promotora de melhores práticas neste campo.

\section{Aconselhamento em saúde}

$\mathrm{Na}$ literatura científica, encontramos diferentes definições do aconselhamento psicológico, sumarizadas em livros clássicos da área (COREY, 1983; MORATO, 1999; PATTERSON; EISENBERG, 1988; SCHEEFFER, 1980) e recentemente revisitados a partir de diversas perspectivas teóricas (HUTZ-MIDGETT; HUTZ, 2012). Inicialmente como um campo demarcado por sua origem educacional e organizacional, com forte influência da teoria traço e fator, passou a ser aplicado em diferentes contextos, entre eles o da saúde pública. Atravessando as diversas definições, encontram-se expressões como a relação de ajuda, tecnologia de cuidado, relação interpessoal, relacionamento cliente e profissional, entre outras.

Para a Organização Mundial da Saúde (OMS), o aconselhamento apóia-se no estabelecimento de relações e condições favoráveis para que o indivíduo avalie seus problemas e tome decisões. Para o Ministério da Saúde (1997), trata-se de uma escuta ativa, individualizada, centrada no cliente. Pressupõe a capacidade de estabelecer relações de confiança entre interlocutores, visando ao resgate de recursos internos do cliente para que ele mesmo tenha possibilidade de reconhecer-se como sujeito de sua própria saúde e transformação. Assim, esse processo envolve lidar com problemas de ordem de ordem afetiva, 
reconhecendo potencialidades e recursos internos, em uma proposição inspirada na abordagem centrada na pessoa (ROGERS, 2001), que influenciou fortemente os estudos da área a partir da década de 1940 ao aproximar o aconselhamento da prática psicoterápica (SCORSOLINI-COMIN, 2014).

O aconselhamento em saúde envolve, portanto, a clarificação para o cliente acerca de determinados aspectos da sua condição de saúde, a fim de que a pessoa possa, consciente de seus limites e potencialidades, tomar decisões quanto à sua saúde e ao seu tratamento, buscando sempre uma maior autonomia e crescimento pessoal diante do sofrimento que enfrenta. Cabe ao profissional que desenvolve o aconselhamento - que nem sempre é exclusivamente o psicólogo, mas também o enfermeiro e os agentes comunitários de saúde, por exemplo, acompanhar o cliente nesse processo, oferecendo suporte emocional e buscando ampará-lo nas dificuldades cotidianas relacionadas ao adoecer. O profissional de saúde deve acompanhar o cliente ao longo do processo de adoecimento e de tratamento, estando atento às modificações em seu quadro e também no seu modo de compreender a sua condição e implicar-se no tratamento.

Ainda no campo do aconselhamento em saúde, autores destacam que esse processo envolve a capacidade de avaliação de riscos, reconhecimento de situações de risco, tomada de decisões, troca de informações sobre a doença, prevenção e tratamento, permitindo uma relação educativa diferenciada, como, por exemplo, no contexto do HIV e aids (FILGUEIRAS; DESLANDES, 1999). Em estudo recente, Pupo e Ayres (2013) sumarizam o aconselhamento psicológico na saúde pública como uma tecnologia de ajuda, de cuidado e como prática que oferece auxílio estruturado e personalizado para o manejo de situações difíceis e de crises que exigem ajustamento e adaptações para a solução de problemas específicos e para a tomada de decisões. Assim, trata-se de uma prática que se constitui entre o modelo médico e a educação (SCHMIDT, 2012), dando voz ao cliente em suas dificuldades e sofrimentos, promovendo um espaço de escuta que potencializa a pessoa para a autonomia e a busca do bem-estar. Ao destacar-se a terminologia tecnologia de cuidado, expressa-se um dos focos do aconselhamento, que é justamente na intervenção, na prática, na composição de um repertório de ações e estratégias que podem contribuir para o estabelecimento e a manutenção da relação interpessoal e educativa com vistas à promoção da saúde.

Como forma de verificar o status da produção científica atual na área do aconselhamento, foi realizado um levantamento nas bases Lilacs, SciELO, PePSIC e PsycINFO, de 2000 a 2013, recuperando apenas artigos que retratassem práticas e pesquisas na área. A partir dos critérios de inclusão e exclusão adotados, foram recuperados na íntegra 
44 produções, sendo 33 em inglês. A maioria é proveniente dos Estados Unidos $(n=30)$, seguida pelo Brasil $(n=9)$ e publicada nos últimos cinco anos $(n=16)$. O periódico mais frequente é o Journal of Counseling Psychology $(n=18)$. Em termos do perfil dos estudos, predominam as pesquisas de corte transversal e de abordagem qualitativa $(n=16)$, empíricas $(n=23)$, desenvolvidas em serviços-escolas de Psicologia $(n=16)$, com estudantes universitários $(\mathrm{n}=18)$ e com o emprego de questionários e escalas $(\mathrm{n}=12)$. Por esse levantamento (SCORSOLINI-COMIN, 2015a), deflagra-se algumas necessidades na área, como a constante avaliação dos programas de formação em aconselhamento, necessidade de diferenciá-lo de outras práticas (como a psicoterapia), refinamento teórico em contraposição ao relato de experiência e, por fim, levantamento de diferentes metodologias de pesquisa na área. Ainda não são expressivas as produções no campo da saúde pública, o que nos faz atentar para o compartilhamento de saberes, ou seja, para a necessidade de um trabalho multi e interdisciplinar que possa fazer uso de algumas técnicas do aconselhamento psicológico para a promoção da saúde e do bem-estar, o que pode estar presente no modo como diferentes profissionais de saúde lidam com seus clientes no seu cotidiano, de modo que a escuta não seja uma ferramenta exclusiva do profissional de Psicologia insePOR rido neste contexto. Outra revisão, especificamente com foco na pós-graduação brasileira (SCORSOLINICOMIN; SANTOS, 2013) apontou para a necessidade de mais pesquisas e intervenções em instituições extramuros, abrindo espaço para algumas considerações no campo da saúde pública.

\section{Aconselhamento na saúde pública}

No contexto brasileiro, a saúde pública tem sido debatida tendo em vista um denso processo de mudanças que ocorreram a partir do processo de redemocratização do país, da busca por uma reforma sanitária ao final do século XX, da assunção da Reforma Psiquiátrica, da inserção do direito à saúde como responsabilidade do Estado a partir da Constituição Federal de 1988 e do surgimento do Sistema Único de Saúde (SUS) como uma das principais inovações da reforma do Estado brasileiro (BARROSO; SILVA, 2011; DIMENSTEIN, 2001; NASCIMENTO; SCORSOLINI-COMIN; PERES, 2013). A esses eventos acrescenta-se a VIII Conferência Nacional de Saúde em 1986 e diversas discussões e fóruns nos quais se debatia, entre outros, a atuação do profissional de Psicologia neste contexto em mutação. Todo esse movimento na saúde pública revela um gradual processo de democratização no acesso a tratamentos e intervenções, com priorização da atenção básica, ou seja, que atua não no nível remediativo, mas preventivo e de promoção de saúde. Ao promover saúde, afasta-se 
a possibilidade de adoecimento e amplia-se a educação em saúde, que envolve práticas voltadas para o cuidado e para a adoção de atitudes relacionadas ao bem-estar.

Assim, é importante reforçar os aspectos históricos, sociais e políticos desse momento, que contribuíram para a organização de um novo modelo de saúde pública voltado não mais para a doença, mas para um conceito mais amplo e complexo de saúde. A ausência de doenças como sinônimo de saúde foi substituída, neste novo paradigma, por uma noção de bem-estar físico, psicológico, emocional, social e espiritual no qual o sujeito realiza suas capacidades e, segundo a OMS, pode fazer face ao estresse normal da vida, trabalhar de forma produtiva e contribuir para a sociedade em que se insere.

Compreendendo, pois, a saúde também como um processo histórico e social, a inserção dos psicólogos nas políticas públicas de saúde ocorreu ao final da década de 1970. Em 2006, a ABEP (Associação Brasileira de Ensino de Psicologia), em parceria com o MEC e com a OPAS (Organização Pan-americana de Saúde), realizou a Oficina Nacional da ABEP com o tema Psicologia e Saúde Pública/Coletiva. Discute-se, nesse cenário, que a atuação do psicólogo deve considerar o processo de cidadanização e de constituição do sujeito como capaz de ação e proposição. Este profissional também deve romper o corporativismo, as práticas isoladas e identidade profissional hegemônica ligada à psicoterapia (DIMENSTEIN, 2001; RIBEIRO; LUZIO, 2008).

É nesse sentido que o aconselhamento em saúde deve ser pensado (SCORSOLINICOMIN, 2015b) como uma prática integradora a serviço de um trabalho multi e interdisciplinar, com foco na promoção da saúde, e não ligado diretamente à psicoterapia, o que seria uma prática exclusiva do psicólogo (SCORSOLINI-COMIN, 2014). Em que pesem as aproximações e distanciamentos entre esses campos - do aconselhamento e da psicoterapia, postula-se que o primeiro envolva processos comunicacionais e relacionais que possam ser conduzidos por outros profissionais de saúde em suas práticas cotidianas.

Assim, um agente comunitário de saúde, em suas visitas domiciliares, por exemplo, pode sim oferecer um espaço de escuta qualificado para aquela família que se apresenta. Ele não irá realizar uma psicoterapia, mas utilizará atitudes como a empatia e a autenticidade, consideradas facilitadoras para o estabelecimento da relação de ajuda (ROGERS, 2001), bem como a atenção concentrada, para apoiar a pessoa diante de uma dificuldade, para informá-la acerca de algum processo de saúde-doença e ajudá-la a ter maior autonomia diante de uma decisão relacionada ao seu domínio de atuação. Muitas vezes, o agente comunitário de saúde é o único profissional de saúde que, efetivamente, consegue estar com essa família, sendo que isso constitui uma potência em termos da prevenção de doenças e da promoção de saúde. 
Determinados aspectos do aconselhamento em saúde, desse modo, podem ser aplicados por esses profissionais, a fim de ampliar a conscientização sobre a necessidade de exames preventivos para o câncer de mama e de colo de útero, por exemplo, como tem sido desenvolvido em algumas experiências conduzidas pela Estratégia Saúde da Família (SANTOS, 2014). Esses profissionais de saúde, priorizados na atenção básica, são veículos importantes para a oferta de ajuda e para o esclarecimento de dúvidas, bem como para a veiculação de práticas educativas voltadas para a promoção do bem-estar.

O aconselhamento em saúde pode fazer parte do processo de formação desses profissionais, em parceria, por exemplo, com os psicólogos. Essas parcerias tornam-se possíveis em alguns programas como os de Residência Multiprofissional em Saúde, em que são descritas experiências exitosas com diferentes profissionais que atuam na atenção básica (SERRALHA; CHAPADEIRO, 2014). Em todas essas intervenções, há que se primar pelos princípios éticos, como a confidencialidade, haja vista que o setting do atendimento, na maioria das vezes, é composto pelo domicílio do cliente. Assim, a reflexão ética deve fazer parte da formação e do exercício profissional como forma de proporcionar um clima de aceitação e confiança para que as pessoas possam expor suas problemáticas ou sofrimentos relacionados ao processo saúde-saúde (DINIZ; GUEDES, 2005).

O aconselhamento apresenta-se, também, como uma prática no contexto da educação para a saúde, ou seja, de um conjunto de saberes e práticas orientados para a prevenção de doenças e promoção da saúde. A atenção básica, desse modo, constitui um contexto privilegiado para a ampliação da compreensão do processo saúde-doença e da busca por relações mais horizontalizadas. No domínio da saúde pública, o profissional de Psicologia e que desenvolve o aconselhamento psicológico deve ser capaz de propiciar espaço para o cliente expressar o que sabe, pensa e sente. Também deve responder a dúvidas e necessidades, prestando apoio emocional. Para tanto, deve desenvolver uma comunicação competente, fornecendo informações apropriadas às necessidades do usuário e adequadas (e corretas) do ponto de vista científico. Deve empregar linguagem adequada e informações sobre riscos a partir das vivências do usuário (FILGUEIRAS; DESLANDES, 1999). Essas orientações devem deveriam atravessar a formação dos demais profissionais de saúde, pois essas ferramentas de comunicação estão presentes no fazer de diferentes profissionais. Assim, o aconselhamento pode ser incorporado na formação mais ampla em saúde, sendo o psicólogo um auxiliar nesse processo de desenvolver e discutir o estabelecimento da relação de ajuda e os meandros da comunicação entre profissionais e clientes. 
Essas orientações para o aconselhamento em saúde são observadas, portanto, a partir da implementação da estratégia saúde da família (ESF) como forma de contribuir com a construção e consolidação dos princípios do SUS: universalidade, equidade e integralidade. Como o apoio de equipamentos como o NASF a partir de 2008, a atenção básica tem buscado aumentar a resolutividade, reforçando os processos de territorialização e regionalização em saúde, oferecendo suporte matricial (SANTEIRO, 2012). Assim, a ESF visa a descentralizar a gestão administrativa, fortalecendo os municípios dentro dos princípios do SUS, reafirmando a atuação de equipes profissionais centradas na perspectiva coletiva e comprometida com os direitos sociais. O foco está na prevenção de doenças e na promoção da saúde, em um trabalho que perpassa a atuação com grupos e a gestão por equipes (ANDRADE; SIMON, 2009; CAMARGO-BORGES; CARDOSO, 2005; CAMARGO-BORGES; MISHIMA, 2009; SPINK, 2010). Abre-se cada vez mais espaço para a humanização como estratégia de interferência no processo de produção em saúde. O pressuposto é o de que sujeitos, quando mobilizados, são capazes de transformar realidades, transformando-se a si próprios no mesmo processo (BENEVIDES; PASSOS, 2005). Humanizar, para esses autores, é qualificar as práticas em saúde, promovendo acesso com acolhimento, atenção integral e equânime com responsabilização e vínculo. Há que se primar pela valorização dos trabalhadores e usuários com avanço na democratização dos processos de gestão e de participação social.

Esse paradigma em saúde, portanto, parte de uma reorientação do modelo assistencial, o que deve ser abarcado pelos profissionais de Psicologia que compõem as equipes de saúde na ESF, nos NASF e em demais equipamentos ligados à saúde pública. A ESF, como destacado pela OPAS, envolve um contínuo "fazer" e "refazer", o que evidencia a necessidade que os profissionais analisem crítica e constantemente as suas práticas (CAMARGO-BORGES; CARDOSO, 2005).

A pesquisa também é fundamental nesse processo, pois pode subsidiar a adoção de novas e diferentes práticas (SPINK, 2010). Obviamente que essas pesquisas devem estar articuladas com o universo das práticas, incentivando não apenas que experiências bemsucedidas sejam relatadas, mas que essas intervenções sejam avaliadas e discutidas, com espaço para a experimentação de orientações e reinvenções que envolvem o acolhimento, o cuidado e a possibilidade de ressignificação do sujeito. Pelas lacunas observadas na literatura científica sobre o aconselhamento, destaca-se a necessidade de mais pesquisas extramuros que possuam os equipamentos de saúde não apenas como contextos de produção, mas como espaço de reflexão e de construção de conhecimento (SCORSOLINI-COMIN; SANTOS, 2013). 
Pesquisas de corte longitudinal, embora sejam mais complexas de serem realizadas, podem oferecer suporte para estudos sobre mudança terapêutica, avanços e retrocessos nos tratamentos oferecidos, efetividade das técnicas de aconselhamento a longo prazo, considerando o papel das diversas transições ao longo do tempo. Pesquisas mistas, com delineamento quanti-quali também podem contribuir para estudos de perfil, por exemplo, de uma dada clientela de um serviço de saúde, contemplando não apenas os aspectos que podem ser generalizados para outras situações como também as nuanças e particularidades do contato profissional, da relação de ajuda estabelecida entre cliente e profissional (HANSON et al., 2005). As pesquisas internacionais encontram-se nesse movimento, o que destaca a necessidade de que os estudos nacionais sejam cada vez mais enriquecidos com a diversidade de técnicas, saberes e referenciais.

\section{A Psicologia Positiva na saúde pública e a formação do profissional de Psicologia}

Neste sentido, apresenta-se o referencial da Psicologia Positiva como alternativa para práticas e pesquisas orientadas para o bem-estar e, portanto, compatíveis com a perspectiva de promoção da saúde no domínio da saúde pública. O movimento da Psicologia Positiva, embora tenha se revelado academicamente na década de 1990, a partir dos trabalhos de Seligman (2011), possui sua origem na década de 1950 nos estudos de Rogers e Maslow. Maslow foi quem cunhou a expressão Psicologia Positiva. Trata-se de uma abordagem cujo foco recai na busca do bem-estar, de fortalecimento dos aspectos positivos do ser humano, com destaque para os recursos desenvolvidos para o enfrentamento de situações adversas. Surge em um contexto no qual o foco estava no estudo exclusivo das doenças e das psicopatologias, com pouco destaque para os aspectos adaptativos e para a dimensão do que promove o bem-estar.

Seligman (2011), por exemplo, acredita que o bem-estar envolve o engajamento pessoal, a busca por um sentido de vida e a existência de relacionamentos interpessoais que promovam a assunção de afetos positivos. O foco, novamente, está na promoção de saúde e, para além desta, na potencialização (BARROS; MARTÍN; PINTO, 2010; PALUDO; KOLLER, 2007; SCORSOLINI-COMIN; FONTAINE; KOLLER; SANTOS, 2013; SELIGMAN; CSIKSZENTMIHALYI, 2000; SNYDER; LOPEZ, 2009).

O modelo criado por Fredrickson (2001, 2009), conhecido como "construir e potencializar" ou "ampliar e potencializar" abarca a intervenção em quatro momentos: (a) prevenção primária, quando o adoecimento ainda não ocorreu; (b) prevenção secundária, quando o adoecimento foi diagnosticado e a intervenção busca prevenir novos sintomas ou 
repercussões; (c) potencialização primária, que consiste em atividades para estabelecer a satisfação e o funcionamento ótimos; (d) potencialização secundária, que partem de quando o funcionamento e a satisfação já estão bons para atingir experiências de pico. Neste enfoque, portanto, a promoção de saúde pode se dar a partir das potencializações orientadas em um modelo de intervenção que abre espaço para a escuta dos aspectos apreciativos, ou seja, não apenas para o adoecimento como também para os recursos trazidos pelo cliente e que podem ser desenvolvidos e aprimorados, por exemplo, em um processo de aconselhamento psicológico de base positiva (SCORSOLINI-COMIN, 2014).

Embora alguns desses aspectos sejam cotejados por outras abordagens (entre elas o humanismo e o construcionismo social), o que se aponta é o modo como essas experiências de bem-estar são priorizadas não apenas para acessar o cliente, mas para fortalecer vínculos e buscar o chamado "florescimento". Este conceito é a condição que permite o desenvolvimento saudável e positivo dos aspectos biológicos, psicológicos e sociais dos seres humanos (SELIGMAN, 2011), que também dialoga com a busca ou a tendência para a autorrealização em Rogers (2001), também em busca de crescimento, maturidade, autonomia e aprendizagem significativa. A busca pelo bem-estar, com um dos pilares da Psicologia Positiva, recupera a necessidade de que sejam fortalecidas não apenas as vinculações sociais, mas aspectos relacionados à própria experiência subjetiva de satisfação com a vida. Ampliando os afetos positivos (por exemplo, otimismo, entusiasmo, esperança) e ajudando o cliente a reconhecer as experiências de bem-estar em sua vivência cotidiana pode-se contribuir para que o indivíduo se posicione de modo mais adequado diante de situações consideradas adversas, como o enfrentamento do adoecimento. Estando aberto para as experiências positivas, como as de aprendizagem, pode-se fortalecer que experiências de dor e sofrimento possam trazer, também, amadurecimento e uma maior potência para o enfrentamento, evocando os recursos disponíveis (SCORSOLINI-COMIN; SANTOS, 2010; SELIGMAN, 2011).

O aconselhamento em saúde deve focalizar, tendo como norte a alternativa da Psicologia Positiva, não apenas o relato do sofrimento, mas os efeitos desses relatos nas práticas que serão desenvolvidas pelo cliente posteriormente - entre esses efeitos destacamos a aprendizagem, a transferência de conhecimentos para a comunidade, a possibilidade de reagir de modo mais adequado diante de eventos semelhantes, o cuidado, a busca por uma melhor condição de vida, ou, ainda, a utilização de recursos importantes que proporcionem bem-estar, amadurecimento e instilação de esperança. $\mathrm{O}$ aconselhamento em saúde não pode estar associado a uma "receita" do que o cliente deve fazer ou ser, mas buscar a construção conjunta, na relação de ajuda, de uma forma mais amadurecida e palatável de ser e fazer, 
tendo como circunscritoras as condições de saúde, por exemplo, e como potencializadores os recursos já trazidos pelo cliente ou que podem emergir a partir desses contatos com os profissionais de saúde sensíveis a esse processo. É nesse sentido que a escuta, aqui priorizada sob o prisma do aconselhamento psicológico, pode ser compreendida como produtora de saúde, justamente por ativar e desenvolver recursos, cuidado e formas de ser que estejam alinhadas a uma postura de promoção de saúde, de valorização dos aspectos positivos e de educação como ferramenta de fortalecimento, amadurecimento e difusão de saberes e práticas que visam a ajudar as pessoas nas mais variadas situações de vida em comunidade.

Por fim, ao falarmos em pesquisa e prática no campo da saúde pública, precisamos abordar a formação profissional e o espaço de criação e recriação de práticas e saberes a partir de experiências de estágio. Por destacarmos neste estudo o aconselhamento psicológico, abordaremos especificamente a formação em Psicologia (BOARINI, 1996; SCORSOLINICOMIN; SOUZA; SANTOS, 2008). Os estágios supervisionados em Psicologia, divididos em básicos e específicos, devem possibilitar aos estudantes o contato com a diversidade, com os múltiplos referenciais e com os diferentes campos, entre os quais destacamos, neste estudo, a saúde pública. O estágio básico foi regulamentado em 2004 pelas Diretrizes Curriculares Nacionais para os cursos de Psicologia como forma de integrar os conteúdos adquiridos no ciclo básico de formação a partir da inserção no universo da prática, o que pode ocorrer de diferentes formas. Em 2011 essas diretrizes foram atualizadas para incorporar orientações para a formação do professor de Psicologia. A atuação em saúde, a partir desse documento, deve se comprometer com ações de prevenção de doenças e promoção de saúde, com vistas à proteção e reabilitação da saúde psíquica e psicossocial. Trata-se de enfatizar, desde os estágios básicos, a promoção da qualidade de vida, a valorização da atenção básica e do trabalho em equipe, retomando os princípios balizados nas políticas públicas em vigência na área da saúde.

A formação em Psicologia, desde os estágios básicos, que devem ocorrer já nos primeiros semestres letivos, acena para as potencialidades do emprego do aconselhamento psicológico não apenas para instrumentalizar a aproximação com o campo empírico e para que o estudante entre em contato com a literatura sobre a primeira entrevista, a entrevista de ajuda, as atitudes facilitadoras, o processo terapêutico ou as interfaces que envolvem o relacionamento entre profissional e cliente. Para além dos conhecimentos e treinamentos a partir de técnicas que envolvem a escuta, o acolhimento, a ética e o comprometimento em uma relação de ajuda, o aconselhamento psicológico no estágio básico deve promover uma reflexão do estudante a partir do seu "fazer" e "refazer" (CAMARGO-BORGES; CARDOSO, 
2005), promovendo a reconstrução da subjetividade do futuro profissional do campo da saúde, erradicando, nas palavras de Dimenstein (2001), o desinteresse, a alienação e o agir mecânico, com fortalecimento da capacidade de agir e refletir. É desse modo que se pode pensar, de fato, em uma prática de "fronteira" (SCHMIDT, 2012), construída entre o modelo médico e a educação, contextualizada, atenta aos movimentos históricos, sociais e que dizem respeito ao humano.

Assim, o aconselhamento se constitui como um campo de constante reinvenção de práticas, por exemplo, quando apresenta à comunidade a modalidade do plantão psicológico (MAHFOUD, 2012; PERCHES; CURY, 2013), já consagrado em nosso país e que articula os saberes da saúde, da psicoterapia e da atuação na comunidade. O plantão psicológico, muito empregado como uma das primeiras aproximações práticas do estudante no estágio básico em Psicologia, pode e deve favorecer o contato do aluno com uma escuta fina, qualificada e atenta aos movimentos de autonomia do cliente e de potencialização de seus recursos. Mas, ao mesmo tempo, como espaço de criação, inovação, atento às competências multiculturais que enfocam o papel da cultura, dentro da proposta de um cuidado que dialogue não apenas com a dimensão do indivíduo (o que, por vezes, tem sido priorizado na prática e na pesquisa em Psicologia), mas também com o seu meio, com a sua cultura e com os diversos contextos nos quais transita e que não constituem apenas cenários fixos, mas prenhes de significado (SEHGAL et al., 2011).

\section{Considerações finais}

A partir dessas considerações, podemos concluir que o aconselhamento psicológico em saúde pública apresenta diversas possibilidades tanto na prática quanto na pesquisa, sendo esta última ainda uma lacuna a ser preenchida pelos pesquisadores da área. A aproximação com a Psicologia Positiva, neste sentido, pode ser um convite não apenas para o delineamento e a utilização de intervenções de base positiva junto a diferentes equipamentos de saúde, mas para pesquisas conduzidas neste contexto, o que pode e deve estar atrelado à avaliação dos programas existentes. Orientar positivamente uma intervenção em aconselhamento reflete uma ação que efetivamente valoriza o recurso para além do foco exclusivo na queixa, no sintoma, nos afetos negativos e nos aspectos que não são adequados e promotores de bemestar. $\mathrm{O}$ aconselhamento nesta abordagem justamente se mostra aberto à escuta dos recursos, dos aspectos preservados, das potências e virtudes trazidas por cada cliente, independentemente de seu quadro e de suas condições de vida. 
As práticas profissionais devem se alinhar cada vez mais à promoção da saúde como espaço para o desenvolvimento do sujeito em um paradigma que repensa constantemente as ações, com abertura para o necessário diálogo entre abordagens e fazeres. A pesquisa deve ser compreendida como parte indissociável desse diálogo. Ou, nas palavras de Bakhtin (1999), que "duas vozes são o mínimo de vida", o que recupera a prática e a pesquisa como dimensões que constituem, estruturam e possibilitam a emergência de uma saúde pública com o seu devido compromisso com o humano e o social.

Este estudo teórico nos convida para a apreciação da Psicologia Positiva como vertente teórica que pode orientar os processos de aconselhamento em saúde visando à promoção do bem-estar como cerne do desenvolvimento humano. Se o bem-estar constitui um dos principais objetivos da Psicologia Positiva, há que se compreender de que modo a prática do aconselhamento pode favorecer essa busca - e esta experiência - nos contextos de saúdedoença. A atenção primária constitui um locus importante nesta reflexão por já se estruturar em um modelo no qual a prioridade é a promoção de saúde, a prevenção e a educação. A escuta, muitas vezes representada como um momento de confiança, entrega, disponibilidade e de criação de caminhos possíveis, pode ser um espaço de produção de saúde justamente por valorizar narrativas de bem-estar, recuperação, superação e de reconstrução. Desse modo, propõe-se esta escuta como prática em saúde e que deve atravessar constantemente as ações dos diferentes profissionais inseridos não apenas na atenção primária, mas também em todo o sistema de saúde pública. Espera-se que tais reflexões possam disparar o diálogo e a escuta para as diversas possibilidades que emergem desse olhar para a saúde.

\title{
COUNSELING PSYCHOLOGY AND POSITIVE PSYCHOLOGY IN PUBLIC HEALTH: LISTENING AS HEALTH PRODUCTION
}

\begin{abstract}
:
The objective of this theoretical study is to present and discuss the Counseling Psychology in the field of public health as a way to provide a listening while production of health, ie, assuming that the space created for talks about the problems experienced already a tool capable of producing well-being. Positive Psychology is brought as a suitable approach to the assumptions of public health and counseling focus on the field of health in understanding the human being, his virtues and strengths. Reflecting on professional training in psychology in the field of public health, counseling is also presented as a possible inclusion in this scenario the trainee and significant about the competencies and skills that must be going through and be professional learning. It is concluded that listening can be valued by health professionals as a way to bring them closer to customers and enhance the development and recognition of resources for the promotion of well-being.
\end{abstract}

Keywords: Counseling Psychology; public health; Positive Psychology. 


\section{ASESORÍA PSICOLÓGICA Y PSICOLOGÍA POSITIVA EN SALUD PÚBLICA: EL ESCUCHAR COMO LA PRODUCCIÓN DE SALUD}

\section{Resumen:}

El objetivo de este estudio teórico es presentar y discutir la orientación psicológica en el campo de la salud pública como una manera de proporcionar una escucha mientras que la producción de la salud, es decir, suponiendo que el espacio creado para el habla de los problemas que ya experimentó una herramienta capaz de producir el bienestar. Psicología Positiva es llevada como un enfoque adecuado a los supuestos de la salud pública y el asesoramiento se centran en el campo de la salud en la comprensión del ser humano, sus virtudes y fortalezas. Al reflexionar sobre la formación profesional en psicología en el campo de la salud pública, el asesoramiento también se presenta como una posible inclusión en este escenario el aprendiz y significativa acerca de las competencias y habilidades que deben estar pasando por y ser profesional de aprendizaje. Se concluye que la escucha puede ser valorado por los profesionales de la salud como una manera de acercarlos a los clientes y mejorar el desarrollo y el reconocimiento de los recursos para la promoción del bienestar.

Palabras clave: asesoramiento psicológico; salud pública; Psicología Positiva.

\section{Referências}

ANDRADE, J. F. S. M.; SIMON, C. P. Psicologia na atenção primária à saúde. Paidéia, Ribeirão Preto, v. 19, n. 43, p. 167-175, 2009.

BAKHTIN. M. Marxismo e filosofia da linguagem. São Paulo: Hucitec, 1999.

BARROS, R. M. A.; MARTíN, J. I. G.; PINTO, J. F. V. C. Investigação e prática em Psicologia positiva. Psicologia: Ciência e Profissão, Brasília, v. 30, n. 2, p. 318-327, 2010.

BARROSO, S. M.; SILVA, M. A. Reforma psiquiátrica brasileira: o caminho da desinstitucionalização pelo olhar da historiografia. Revista da SPAGESP, Ribeirão Preto, v. 12, n. 1, p. 66-78, 2011.

BENEVIDES, R.; PASSOS, E. Humanização na saúde: um novo modismo? Interface, Botucatu, v. 9, n. 17, p. 389-394, 2005.

BOARINI, M. L. A formação (necessária) do psicólogo para atuar na saúde pública. Psicologia em Estudo, Maringá, v. 1, n. 1, p. 93-132, 1996.

BRASIL. Manual de controle das doenças sexualmente transmissíveis. Ministério da Saúde. Brasília, DF: Coordenação Nacional de DST/Aids, 1997.

CAMARGO-BORGES, C.; CARDOSO, C. L. A Psicologia e a estratégia saúde da família: compondo saberes e fazeres. Psicologia \& Sociedade, v. 17, n. 2, p. 26-32, 2005.

CAMARGO-BORGES, C.; MISHIMA, S. M. A responsabilidade relacional como ferramenta útil para a participação comunitária na atenção básica. Saúde e Sociedade, v. 18, n. 1, p. 29 41, 2009. 
CONSELHO NACIONAL DE EDUCAÇÃO [CNE]. Resolução CNE/CES $n^{\circ} 8$, de 7 de maio de 2004: institui as Diretrizes Curriculares Nacionais para os cursos de graduação em Psicologia. Recuperada de < http://portal.mec.gov.br/cne/arquivos/pdf/rces08_04.pdf>. . Resolução CNE/CES n ${ }^{\circ}$ 5, de 15 de março de 2011: institui as Diretrizes Curriculares Nacionais para os cursos de graduação em Psicologia, estabelecendo normas para o projeto pedagógico complementar para a Formação de Professores de Psicologia. Recuperada de < http://portal.mec.gov.br/index.php?option=com_docman\&task=doc_download\&gid=7692\&It emid=.>.

COREY, G. Técnicas de aconselhamento e psicoterapia. Trad. M. L. E. Silva. Rio de Janeiro: Campus, 1983.

DIMENSTEIN, M. O psicólogo e o compromisso social no contexto da saúde coletiva. Psicologia em Estudo, Maringá, v. 6, n. 2, p. 57-63, 2001.

DINIZ, D.; GUEDES, C. Confidencialidade, aconselhamento genético e saúde pública: um estudo de caso sobre o traço falciforme. Cadernos de Saúde Pública, v. 21, n. 3, p. 747-755, 2005.

FILGUEIRAS, S. L.; DESLANDES, S. F. Avaliação das ações de aconselhamento: análise de uma perspectiva de prevenção centrada na pessoa. Cadernos de Saúde Pública, v. 15, supl. 2, p. 121-31, 1999.

FREDRICKSON, B. L. The role of positive emotions in positive psychology: The broadenand-built theory of positive emotions. American Psychologist, v. 56, p. 218-226, 2001. Positividade: descubra a força das emoções positivas, supere a negatividade e viva plenamente. Trad. P. Libânio. Rio de Janeiro: Rocco, 2009.

HANSON, W. E.; CRESWELL, J. W.; CLARK, V. L. P.; PETSKA, K. S.; CRESWELL, J. D. Mixed methods research designs in Counseling Psychology. Journal of Counseling Psychology, v. 52, n. 2, p. 224-235, 2005.

HUTZ-MIDGETT, A.; HUTZ, C. S. Counseling in Brazil: Past, present, and future. Journal of Counseling \& Development, v. 90, n. 2, p. 238-242, 2012.

MAHFOUD, M. (Org.). Plantão psicológico: novos horizontes. São Paulo: Companhia Ilimitada, 2012.

MORATO, H. T. P. Aconselhamento psicológico: uma passagem para a transdisciplinaridade. In:___. (Orgs.), Aconselhamento psicológico centrado na pessoa: novos desafios. São Paulo: Casa do Psicólogo, 1999, p. 61-90.

MORENO, D. M. F. C.; REIS, A. O. A. Revelação do diagnóstico da infecção pelo HIV no contexto do aconselhamento: a versão do usuário. Temas em Psicologia, Ribeirão Preto, v. 21, n. 3, p. 591-609, 2013.

NASCIMENTO, G. C. M.; SCORSOLINI-COMIN, F.; PERES, R. S. Mental health in the Unified Health System: mapping the contributions from the Psychosocial Care

Centers. SMAD - Revista Eletrônica Saúde Mental Álcool e Drogas, Ribeirão Preto, v. 9, n. 2, p. $95-102,2013$. 
PALUDO, S. S.; KOLLER, S. H. Psicologia Positiva: Uma nova abordagem para antigas questões. Paidéia, Ribeirão Preto, v. 17, n. 36, p. 9-20, 2007.

PATTERSON, L. E.; EISENBERG, S. O processo de aconselhamento. São Paulo: Martins Fontes, 1988.

PEQUENO, C. S.; MACÊDO, S. M.; MIRANDA, K. C. L. Aconselhamento em HIV/AIDS: pressupostos teóricos para uma prática clínica fundamentada. Revista Brasileira de Enfermagem, v. 66, n. 3, p. 437-441, 2013.

PERCHES, T. H. P.; CURY, V. E. Plantão psicológico em hospital e o processo de mudança psicológica. Psicologia: Teoria e Pesquisa, Brasília, v. 29, n. 3, p. 313-320, 2013.

PUPO, L. R.; AYRES, J. R. C. M. Contribuições e limites do uso da abordagem centrada na pessoa para a fundamentação teórica do aconselhamento em DST/Aids. Temas em Psicologia, Ribeirão Preto, v. 21, n. 3, p. 1089-1106, 2013.

RIBEIRO, S. L.; LUZIO, C. A. As diretrizes curriculares e a formação do psicólogo para a saúde mental. Psicologia em Revista, Belo Horizonte, v. 14, n. 2, p. 203-220, 2008.

ROGERS, C. R. Tornar-se pessoa. São Paulo: Martins Fontes, 2001.

SANTEIRO, T. V. Processos clínicos em Núcleos de Apoio à Saúde da Família/NASF: estágio supervisionado. Psicologia: Ciência e Profissão, Brasília, v. 32, n. 4, p. 942-955, 2012.

SANTOS, A. K. M. Aconselhamento em saúde e plano de intervenção para obtenção de maior adesão das usuárias da ESF do município Couto de Magalhães de Minas aos exames de prevenção do câncer de colo de útero. Monografia não publicada. Especialização em Atenção Básica em Saúde da Família, Universidade Federal de Minas Gerais, Belo Horizonte, 2014.

SCHEEFFER, R. Aconselhamento psicológico: teoria e prática. $7^{\mathrm{a}}$ ed. São Paulo: Atlas, 1980.

SCHMIDT, M. L. S. O nome, a taxonomia e o campo do aconselhamento psicológico. In: MORATO, H. T. P.; BARRETO, C. L. B. T.; NUNES, A. P. (Coords.), Aconselhamento psicológico numa perspectiva fenomenológica existencial. Rio de Janeiro, Guanabara Koogan, 2012, p. 1-21.

SCORSOLINI-COMIN, F. Aconselhamento psicológico: práticas e pesquisas nos contextos nacional e internacional. Revista Mal-Estar e Subjetividade, v.

15, n. 1, p. 130-141, 2015 (a).

SCORSOLINI-COMIN, F. Aconselhamento psicológico: Aplicações em gestão de carreiras, educação e saúde. São Paulo: Atlas, 2015 (b). 200 p.

SCORSOLINI-COMIN, F. Aconselhamento psicológico e psicoterapia: aproximações e distanciamentos. Contextos Clínicos, São Leopoldo, v. 7, n. 1, p. 2-14, 2014. 
; FONTAINE, A. M. G. V.; KOLLER, S. H.; SANTOS, M. A. From authentic happiness to well-being: the flourishing of Positive Psychology. Psicologia: Reflexão $e$ Crítica, Porto Alegre, v. 26, n. 4, p. 663-670, 2013.

; SANTOS, M. A. The scientific study of happiness and health promotion: An integrative literature review. Revista Latino-Americana de Enfermagem, Ribeirão Preto, v. 18, n. 3, p. 472-479, 2010.

. Counseling Psychology: A view of the scientific production in the Brazilian postgraduation. Revista Brasileira de Crescimento e Desenvolvimento Humano, São Paulo, v. 23, n. 3, p. 338-345, 2013.

; SOUZA, L. V.; SANTOS, M. A. Tornar-se psicólogo: Experiência de estágio de Psico-oncologia em equipe multiprofissional de saúde. Revista Brasileira de Orientação Profissional, Ribeirão Preto, v. 9, n. 2, p. 113-125, 2008.

SEHGAL, R.; SAULES, K.; YOUNG, A.; GREY, M. J.; GILLEM, A. R.; NABORS, N. A. Practicing what we know: multicultural counseling competence among clinical Psychology trainees and experienced multicultural psychologists. Cultural Diversity and Ethnic Minority Psychology, v. 17, n. 1, p. 1-10, 2011.

SELIGMAN, M. E. P. Florescer: uma nova compreensão sobre a natureza da felicidade e do bem-estar. Trad. C. P. Lopes. Rio de Janeiro: Objetiva, 2011.

SELIGMAN, M. E. P.; CSIKSZENTMIHALYI, M. Positive psychology: an introduction. American Psychologist, v. 55, p. 5-14, 2000.

SERRALHA, C. A.; CHAPADEIRO, C. A. As famílias que encontramos na atenção básica: Desafios e reflexões para a prática em Psicologia. In: SCORSOLINI-COMIN, F.; SOUZA, L. V.; BARROSO, S. M. (Orgs.), Práticas em Psicologia: Saúde, família e comunidade. Uberaba: Editora da Universidade Federal do Triângulo Mineiro, 2014, p. 305-328.

SNYDER, C. R.; LOPEZ, S. J. Psicologia Positiva: uma abordagem científica e prática das qualidades humanas. Trad. R. C. Costa. São Paulo: Artmed, 2009.

SPINK, M. J. P. Psicologia social e saúde: práticas, saberes e sentidos. Petrópolis: Vozes, 2010.

Data de recebimento: 05/08/2014

Data de aceite: 11/04/2018

\section{Sobre o autor:}

Fabio Scorsolini-Comin é Psicólogo (Bacharelado, Licenciatura e Formação de Psicólogo) pela Faculdade de Filosofia, Ciências e Letras de Ribeirão Preto da Universidade de São Paulo (FFCLRP-USP). Mestre e Doutor em Psicologia pela Universidade de São Paulo (USP). Realizou Doutorado Sanduíche na Faculdade de Psicologia e de Ciências da Educação da Universidade do Porto (Portuga1/Bolsa USP/Santander). Realizou dois Pós-Doutorados na Universidade de São Paulo (2013-2015 e 2016-2017), na área de Tratamento e Prevenção Psicológica (ambos com Bolsa PDJ-CNPq). Docente do Departamento de Enfermagem Psiquiátrica e Ciências Humanas da Escola de Enfermagem de Ribeirão Preto da Universidade de São Paulo - EERP-USP. Endereço Eletrônico: fabio.scorsolini@usp.br 